Pacific Journal of Mathematics

CHARACTERIZATION OF $C^{*}$-ALGEBRAS WITH CONTINUOUS
TRACE BY PROPERTIES OF THEIR PURE STATES 


\title{
CHARACTERIZATION OF $C^{*}$-ALGEBRAS WITH CONTINUOUS TRACE BY PROPERTIES OF THEIR PURE STATES
}

\author{
R. J. ARChbold ANd Frederic W. Shultz
}

\begin{abstract}
We characterize $C^{*}$-algebras with continuous trace among all $C^{*}$ algebras by a condition on the set $P(A)$ of pure states. The condition is that (1) the graph $R(A)$ of the unitary equivalence relation on $P(A)$ is closed in $P(A) \times P(A)$, and (2) transition probabilities are continuous for the product topology on $R(A)$ (i.e. that inherited from $P(A) \times P(A)$ ). If $R(A)$ is given the quotient topology, these conditions are equivalent to properness of the inclusion map from $R(A)$ into $P(A) \times P(A)$. We show the product and quotient topologies on $R(A)$ coincide iff transition probabilities are continuous for the product topology, and this in turn is equivalent to Fell's condition. Transition probabilities are always continuous for the quotient topology on $R(A)$.
\end{abstract}

Introduction. In [15] it was shown that the set $P(A)$ of pure states of a $C^{*}$-algebra $A$ determines $A$ up to *-isomorphism. Here $P(A)$ carries the structure of a uniform space (for the weak* uniformity), transition probabilities (or equivalently, the distance given by the norm on $\left.P(A) \subseteq A^{*}\right)$, and orientation.

In $\S 2$ of the current paper we explore a connection between two of these structures by studying weak* continuity of transition probabilities. Continuity on all of $P(A) \times P(A)$ is rare: it occurs only for $A$ equal to a $c_{0}$ direct sum of elementary $C^{*}$-algebras. (Equivalently: $A$ is type $\mathrm{I}$ and $\hat{A}$ is discrete.) The set $R(A)$ of pairs of unitarily equivalent pure states provides a more interesting domain for transition probabilities. For the topology inherited as a subspace of $P(A) \times P(A)$ (which we call the product topology on $R(A)$ ), continuity of transition probabilities restricted to $R(A)$ is equivalent to Fell's condition. (Roughly following the terminology in [4], we say a $C^{*}$-algebra $A$ satisfies Fell's condition if for each $\pi_{0}$ in $\hat{A}$ there is an element $b$ in $A^{+}$such that $\pi(b)$ is a projection of rank one for all $\pi$ in some neighborhood of $\pi_{0}$.) This gives the characterization of continuous trace algebras described in the abstract above.

There is a second topology on $R(A)$ that is of great interest. Let $G(A)$ be the set of extreme points of the unit ball $A^{*}$. If $\phi$ is in $G(A)$, 
then $\left(|\phi|,\left|\phi^{*}\right|\right)$ is in $R(A)$, and the map $\phi \rightarrow\left(|\phi|,\left|\phi^{*}\right|\right)$ is a continuous surjection onto $R(A)$ with the product topology. In general the product and quotient topologies on $R(A)$ do not agree. The quotient topology played an important role in Renault's applications of the dual groupoid [13].

One virtue of the quotient topology is that $G(A) \rightarrow R(A)$ becomes a locally trivial principal $S^{1}$-bundle, as observed in [13]. Another virtue is discussed in $\S 3$ : transition probabilities are always continuous for the quotient topology on $R(A)$. It is shown that the product and quotient topologies coincide iff transition probabilities are continuous for the product topology. (It follows that these topologies coincide if $A$ has continuous trace; this result is in [11] and [13].) This result can be rephrased by saying $A$ has continuous trace iff the canonical map from $R(A)$ into $P(A) \times P(A)$ is proper. In this form it is closely related to Green's [9] characterization of free actions of groups giving rise to continuous trace algebras: in each case the key property is properness of the map $s \times r$ where $s$ and $r$ are respectively the source and range maps on a principal groupoid.

Finally, we show continuity of transition probabilities at points in the diagonal of $R(A)$ implies continuity on all of $R(A)$ (for the product topology).

Acknowledgment. Some of these results were obtained while the second author was visiting Oxford University. That author wishes to thank Charles Batty and the SERC for making that visit possible.

1. Preliminaries. Let $A$ be a $C^{*}$-algebra. If $\phi$ and $\psi$ are pure states on $A$, let $p$ and $q$ be their respective support projections. Then the transition probability between $\phi$ and $\psi$ is denoted $\langle\phi, \psi\rangle$ and is defined by

$$
\langle\phi, \psi\rangle=\phi(q)=\psi(p) .
$$

If $\phi$ and $\psi$ are unitarily equivalent, there will be an irreducible representation $\pi: A \rightarrow B(H)$ and unit vectors $\xi$ and $\eta$ such that

$$
\phi(a)=(\pi(a) \xi, \xi) \text { and } \psi(a)=(\pi(a) \eta, \eta)
$$

for all $a$ in $A$. Then $\langle\phi, \psi\rangle=|(\xi, \eta)|^{2}$. (If $\phi$ and $\psi$ are inequivalent their transition probability is zero). If $\langle\phi, \psi)=0$ we say $\phi$ and $\psi$ are orthogonal.

We denote by $P(A)$ the set of pure states on $A$, with the weak* topology. The symbol $G(A)$ denotes the set of all extreme points of the unit ball of $A^{*}$, with the weak*-topology. (We call elements of $G(A)$ "pure functionals".) 
For $b$ in $A$ and $\phi$ in $A^{*}$ we define functionals $b \phi$ and $\phi b$ in $A^{*}$ by

$$
(b \phi)(a)=\phi(b a) \text {, and }(\phi b)(a)=\phi(a b) .
$$

We recall the definition of $|\phi|$, following the conventions in [4, §12]. For each $\phi$ in $A^{*}$ there is a unique pair $(u, \psi)$ with $u$ being a partial isometry in $A^{* *}, \psi$ in $A^{*}$ satisfying $\psi \geq 0,\|\psi\|=\|\phi\|, \phi=u \psi$, $\psi=u^{*} \phi$, and with the final projection of $u$ being dominated by the support of $\psi$. The functional $\psi$ is denoted $|\phi|$ and is called the absolute value of $\phi$. The equation $\phi=u|\phi|$ is called the polar decomposition of $\phi$. We note that some authors define $b \phi, \phi b$, and $|\phi|$ differently, so that what they call the absolute value of $\phi$ would be denoted $\left|\phi^{*}\right|$ here, where $\phi^{*}$ is the adjoint of $\phi$, i.e.

$$
\phi^{*}(a)=\phi\left(a^{*}\right)^{-} .
$$

By [5, Lemma 3.5] the map $\phi \rightarrow|\phi|$ is weak* continuous if restricted to the set of $\phi$ in $A^{*}$ of norm one.

The result below describes the set $G(A)$ of pure functionals more explicitly, and describes the map $\phi \rightarrow|\phi|$. We make no claim of originality. Although we know of no explicit reference, it seems likely this is known by many.

Proposition 1.1. The pure functionals on a $C^{*}$-algebra $A$ are precisely those maps $\phi$ of the form

$$
\phi(a)=(\pi(a) \xi, \eta)
$$

for $\pi: A \rightarrow B(H)$ an irreducible representation and for unit vectors $\xi$ and $\eta$. The absolute value of $\phi$ is given by

$$
|\phi|(a)=(\pi(a) \xi, \xi) .
$$

Proof. Suppose first that $\phi$ is any pure functional on $A$, and let $\phi=u|\phi|$ be its polar decomposition. By [1, Theorem 2.1], $\phi$ in $A$ is a pure functional iff $|\phi|$ is a pure state. Let $(\pi, H, \xi)$ be the GNS representation for $|\phi|$. Then

$$
\phi(a)=(u|\phi|)(a)=(\pi(u a) \xi, \xi)=\left(\pi(a) \xi, \pi\left(u^{*}\right) \xi\right) .
$$

If we put $\eta=\pi\left(u^{*}\right) \xi$, then $\phi$ has the specified form.

Conversely, suppose $\phi$ has the form in (1). Let $c$ be the minimal central projection in $A^{* *}$ such that $\pi(c)=1$, so that $\pi: c A^{* *} \rightarrow B(H)$ is a ${ }^{*}$-isomorphism. Let $u$ be the partial isometry in $c A^{* *}$ such that 
$\pi(u)$ has initial space $\mathbf{C} \eta$, final space $\mathbf{C} \xi$, and maps $\eta$ to $\xi$. Let $\psi$ in $A^{*}$ be defined by

$$
\psi(a)=(\pi(a) \xi, \xi) .
$$

Then $\psi$ is a pure state on $A$. By [1, Theorem 2.1], $\phi$ is a pure functional iff $|\phi|$ is a pure state, so we'll be done if we show $\psi=|\phi|$. It is readily verified that $u \psi=\phi, u^{*} \phi=\psi$, and $u u^{*}=$ support projection of $\psi$. Thus $\phi=u \psi$ is the polar decomposition of $\phi$, completing the proof.

Note that for $\phi$ as in equation (1),

$$
\phi^{*}(a)=\phi\left(a^{*}\right)^{-}=(\pi(a) \eta, \xi)
$$

so that $\left|\phi^{*}\right|(a)=(\pi(a) \eta, \eta)$. In particular, $|\phi|$ and $\left|\phi^{*}\right|$ will be equivalent pure states. Conversely any pair of equivalent pure states are of the form $a \rightarrow(\pi(a) \xi, \xi)$ and $a \rightarrow(\pi(a) \eta, \eta)$ and thus coincide with $\left(|\phi|,\left|\phi^{*}\right|\right)$ for some $\phi$ in $G(A)$.

We let $R(A)$ denote the set of pairs of equivalent pure states, i.e. the graph of the unitary equivalence relation on $P(A)$. By the product topology on $R(A)$ we mean the topology as a subspace of $P(A) \times P(A)$.

There is another topology on $R(A)$ that is of interest, namely the quotient topology with respect to the map $G(A) \rightarrow R(A)$ given by $\phi \rightarrow\left(|\phi|,\left|\phi^{*}\right|\right)$. As mentioned previously, this map is continuous from $G(A)$ to $R(A)$ if the latter is given the product topology, so the identity map on $R(A)$ is continuous from the quotient topology to the product topology. However, as we shall see these topologies need not coincide.

Finally, we observe that if $\phi$ and $\psi$ are in $G(A)$, then it follows from Proposition 1.1 that $\left(|\phi|,\left|\phi^{*}\right|\right)=\left(|\psi|,\left|\psi^{*}\right|\right)$ iff $\phi=\lambda \psi$ for some $\lambda$ in $S^{1}$. Thus $R(A)$ with the quotient topology can also be identified as the quotient of $G(A)$ by the natural action of $S^{1}$.

2. Transition probabilities and the product topology. We will use the symbol \langle\rangle to denote the map from $R(A)$ into $\mathbf{R}$ given by transition probabilities, i.e. $(\phi, \psi) \rightarrow\langle\phi, \psi\rangle$. The purpose of this section is to investigate for which $C^{*}$-algebras $A$ this map is continuous if $R(A)$ is given the product topology.

We will need the following result; see [8, Corollary 9], [10, p. 146], and [12, Lemma 2.4]:

LEMMA 2.1. If $\phi$ and $\psi$ are pure states of a $C^{*}$-algebra $A$, then

$$
\|\phi-\psi\|=2(1-\langle\phi, \psi\rangle)^{1 / 2} .
$$

For $\pi \in \hat{A}$ let $S(\pi)$ be the set of those $\sigma$ in $\hat{A}$ such that $\sigma$ and $\pi$ cannot be separated by disjoint open subsets of $\hat{A}$. Since $\hat{A}-S(\pi)$ is open, $S(\pi)$ is a closed subset of $\hat{A}$ containing $\pi$. 
LEMMA 2.2. Let $A$ be a liminal $C^{*}$-algebra such that \langle\rangle is continuous for the product topology. Then for each $\pi$ in $\hat{A}, \pi$ is an isolated point of the subspace $S(\pi)$.

Proof. Let $\pi \in \hat{A}$ and suppose that $\pi$ is not isolated in $S(\pi)$. Let $\xi$ be a unit vector in the Hilbert space $H_{\pi}$ and let $\phi=\omega_{\xi} \circ \pi \in P(A)$. Let $\mathscr{N}$ be a base of weak*-open neighborhoods of zero in $A^{*}$. Given $N \in \mathscr{N}$ choose $M \in \mathscr{N}$ with $M+M \subseteq N$. The canonical image of $(\phi+M) \cap P(A)$ in $\hat{A}$ is an open neighborhood of $\pi[4,3.4 .11]$ and hence contains some element $\sigma$ of $S(\pi)-\{\pi\}$. Thus there exists a unit vector $\eta \in H_{\sigma}$ such that if $\psi=\omega_{\eta} \circ \sigma$ then $\psi \in(\phi+M) \cap P(A)$.

Since $\sigma \in S(\pi)$ and $A$ is liminal, it follows from the construction in [6, pp. 603-4] that there exists $\left(\phi_{N}, \psi_{N}\right) \in R(A)$ such that

$$
\phi_{N} \in \phi+N, \quad \psi_{N} \in \psi+M \subseteq \varphi+N
$$

and $\left\langle\phi_{N}, \psi_{N}\right\rangle \leq 1 / 2$. The nets $\left(\phi_{N}\right)$ and $\left(\psi_{N}\right)$, indexed by $\mathscr{N}$ in the obvious way, are both convergent to $\phi$. However

$$
\left\langle\phi_{N}, \psi_{N}\right\rangle \nrightarrow\langle\phi, \psi\rangle \text {, }
$$

contradicting the continuity hypothesis.

Theorem 2.3. Let $A$ be a $C^{*}$-algebra. Then \langle\rangle is continuous for the product topology on $R(A)$ iff $A$ satisfies Fell's condition.

Proof. Assume $A$ satisfies Fell's condition, and suppose $\left\{\left(\phi_{\alpha}, \psi_{\alpha}\right)\right\}$ is a net in $R(A)$ which converges (in the product topology) to $(\phi, \psi) \in$ $R(A)$. Let $\pi_{\phi}$ be the GNS representation for $\phi$ and $\pi_{\psi}$ for $\psi$. Note that $(\phi, \psi)$ in $R(A)$ means that $\pi_{\phi}$ and $\pi_{\psi}$ represent the same element of $\hat{A}$. Choose $x$ and $y$ in $A^{+}$such that $\pi(x)$ and $\pi(y)$ are projections of rank one for all $\pi$ in a neighborhood $N$ of $\pi_{\phi}$. Kadison's transitivity theorem implies that we can also arrange $\phi(x)=1$ by replacing $x$ by $u^{*} u x$ for a suitable unitary in $\tilde{A}$, and we may similarly arrange $\psi(y)=1$.

Now choose an index $\alpha_{0}$ such that $\alpha \geq \alpha_{0}$ implies that $\phi_{\alpha}$ and $\psi_{\alpha}$ are in the inverse image of $N$ for the canonical map from $P(A)$ onto $\hat{A}$. For each $\alpha \geq \alpha_{0}$ let $\phi_{\alpha}^{\prime}$ and $\psi_{\alpha}^{\prime}$ be the unique pure states equivalent to both $\phi_{\alpha}$ and $\psi_{\alpha}$ and satisfying $\phi_{\alpha}^{\prime}(x)=\psi_{\alpha}^{\prime}(y)=1$. Let $\pi_{\alpha}$ be the GNS representation for $\phi_{\alpha}^{\prime}$. Since $\phi_{\alpha}^{\prime}$ is equivalent to $\phi_{\alpha}$ and $\phi_{\alpha} \rightarrow \phi$, then $\pi_{\alpha} \rightarrow \pi_{\phi}$. Furthermore for $\alpha \geq \alpha_{0}$

$$
\left\langle\phi_{\alpha}^{\prime}, \psi_{\alpha}^{\prime}\right\rangle=\operatorname{tr} \pi_{\alpha}(x y x) .
$$


Since $x \rightarrow \operatorname{tr} \pi(x)$ is constant (and thus continuous) in a neighborhood of $\pi_{\phi}$, and since

$$
0 \leq x y x \leq\|y\| x^{2} \leq\|y\|\|x\| x,
$$

by $[4,4.4 .2]$ the function $\rightarrow \operatorname{tr} \pi(x y x)$ is continuous at $\pi_{\phi}$. By equation (2), it follows that

$$
\left\langle\phi_{\alpha}^{\prime}, \psi_{\alpha}^{\prime}\right\rangle \rightarrow \operatorname{tr} \pi_{\phi}(x y x)=\langle\phi, \psi\rangle .
$$

Finally, since

$$
\left\langle\phi_{\alpha}, \phi_{\alpha}^{\prime}\right\rangle=\phi_{\alpha}(x) \rightarrow \phi(x)=1
$$

by Lemma 2.1 we have $\left\|\phi_{\alpha}-\phi_{\alpha}^{\prime}\right\| \rightarrow 0$. Similarly $\left\|\psi_{\alpha}-\psi_{\alpha}^{\prime}\right\| \rightarrow 0$. Thus

$$
\begin{aligned}
& \left|\left\langle\phi_{\alpha}, \psi_{\alpha}\right\rangle-\left\langle\phi_{\alpha}^{\prime}, \psi_{\alpha}^{\prime}\right\rangle\right| \\
& \quad \leq\left|\left\langle\phi_{\alpha}, \psi_{\alpha}\right\rangle-\left\langle\phi_{\alpha}, \psi_{\alpha}^{\prime}\right\rangle\right|+\left|\left\langle\phi_{\alpha}, \psi_{\alpha}^{\prime}\right\rangle-\left\langle\phi_{\alpha}^{\prime}, \psi_{\alpha}^{\prime}\right\rangle\right| \\
& \quad \leq\left\|\psi_{\alpha}-\psi_{\alpha}^{\prime}\right\|+\left\|\phi_{\alpha}-\phi_{\alpha}^{\prime}\right\| \rightarrow 0
\end{aligned}
$$

which proves that $\left\langle\phi_{\alpha}, \psi_{\alpha}\right\rangle \rightarrow\langle\phi, \psi\rangle$, finishing the proof that \langle\rangle is continuous for the product topology on $R(A)$.

Now suppose \langle\rangle is continuous for the product topology on $R(A)$. We first show $A$ is type $\mathrm{I}$, and then that it is liminal.

Suppose $A$ is not of type I. Let $\pi: A \rightarrow B(H)$ be an irreducible representation such that $\pi(A) \cap K=\{0\}$, where $K$ is the algebra of compact operators on $H$. Let $\omega_{\xi}$ be a vector state on $\pi(A)$. Let $\phi=\omega_{\xi} \circ \pi$ be the associated pure state of $A$, and let $\psi$ be the extension of $\omega_{\xi}$ to the $C^{*}$-algebra $B=\pi(A)+K$ which satisfies $\psi(K)=0$. By [7, Theorem 2] there is a net $\left\{\psi_{\alpha}\right\}$ of vector states on $B$ such that $\psi_{\alpha} \rightarrow \psi$ on $B$. Let $\phi_{\alpha}=\psi_{\alpha} \circ \pi$, and note that $\left\{\phi_{\alpha}\right\}$ is a net in $P(A)$ which converges to $\phi=\psi \circ \pi$. Let $q$ be the rank one projection onto $\mathbf{C} \xi$. Then if $\psi_{\alpha}$ is the vector state corresponding to a unit vector $\xi_{\alpha}$, we have

$$
\left\langle\phi_{\alpha}, \phi\right\rangle=\left|\left(\xi, \xi_{\alpha}\right)\right|^{2}=\psi_{\alpha}(q) \rightarrow \psi(q)=0 .
$$

On the other hand, the net $\left\{\left(\phi_{\alpha}, \phi\right)\right\}$ converges to $(\phi, \phi)$ in $R(A)$ for the product topology, which shows that \langle\rangle is discontinuous in that topology. This contradiction proves that $A$ is of type I.

To show that $A$ is liminal it suffices to show that each point of $\hat{A}$ is closed: [4, 4.7.15] or [6, Theorem 4]. Since $\hat{A}$ has the quotient topology from $P(A)$, we must show each equivalence class in $P(A)$ is closed in $P(A)$. Suppose $\left\{\phi_{\alpha}\right\}$ is a net of equivalent pure states of $A$ which converges to a pure state $\phi$. By continuity of \langle\rangle , since the net which to $(\alpha, \beta)$ assigns $\left(\phi_{\alpha}, \phi_{\beta}\right)$ converges to $(\phi, \phi)$, then $\left\langle\phi_{\alpha}, \phi_{\beta}\right\rangle \rightarrow 1$. From Lemma 2.1, it follows that $\left\|\phi_{\alpha}-\phi_{\beta}\right\| \rightarrow 0$. Thus $\left\{\phi_{\alpha}\right\}$ is a Cauchy 
net for the norm, and so $\left\{\phi_{\alpha}\right\}$ converges in norm to some state $\psi$, so $\left\|\phi_{\alpha}-\phi\right\| \rightarrow 0$. In particular, for $\alpha$ large enough, $\left\|\phi_{\alpha}-\phi\right\|<2$, and so $\phi_{\alpha}$ and $\phi$ are equivalent by [8, Corollary 9]. Thus equivalence classes are closed in $P(A)$, and so $A$ is liminal.

We now establish that Fell's condition holds. Let $\pi \in \hat{A}$. Since $S(\pi)$ is closed, there exists a closed two-sided ideal $J$ of $A$ such that $S(\pi)=(A / J)^{\wedge}$. By Lemma $2.2, \pi$ is isolated in $(A / J)^{\wedge}$ and so there exists a positive element $b$ of norm one in $A / J$ such that $\pi(b)$ is a projection of rank one and $\sigma(b)=0$ for all other $\sigma \in(A / J)^{\wedge}$. Let $A$ be a positive element of $A$ such that $a+J=b$. Using functional calculus if necessary we may arrange that $\|a\|=1$. By lower semicontinuity [4, 3.3.2], $\|\sigma(a)\| \rightarrow 1$ as $\sigma \rightarrow \pi$ in $\hat{A}$. Note that for each $\sigma \in \hat{A}, \sigma(a)$ is a positive compact operator with largest eigenvalue $\|\sigma(a)\|$.

We claim that there exists $\delta \in(0,1)$ and an open neighborhood $U$ of $\pi$ such that, for each $\sigma \in U,\|\sigma(a)\|$ is the only eigenvalue of $\sigma(a)$ greater than $1-\delta$ and furthermore the eigenspace for $\|\sigma(a)\|$ has dimension one. Supposing otherwise, for each $\delta \in(0,1)$, and open neighborhood $U$ of $\pi$ there exists $\pi_{U, \delta} \in U$, orthogonal unit vectors $\xi_{U, \delta}$ and $\eta_{U, \delta}$ in the Hilbert space for $\pi_{U, \delta}$ and real numbers $\lambda_{U, \delta}, \mu_{U, \delta}>1-\delta$ such that

$$
\pi_{U, \delta}(a) \xi_{U, \delta}=\lambda_{U, \delta} \xi_{U, \delta} \quad \text { and } \quad \pi_{U, \delta}(a) \eta_{U, \delta}=\mu_{U, \delta} \eta_{U, \delta} .
$$

We direct the set of all such $(U, \delta)$ by defining $(U, \delta) \geq\left(U^{\prime}, \delta^{\prime}\right)$ if and only if $U \subseteq U^{\prime}$ and $\delta \geq \delta^{\prime}$. Then, writing $\alpha=(U, \xi)$, we have

$$
\pi_{\alpha} \rightarrow \pi, \quad \pi_{\alpha}(a) \xi_{\alpha}=\lambda_{\alpha} \xi_{\alpha}, \quad \pi_{\alpha}(a) \eta_{\alpha}=\mu_{\alpha} \eta_{\alpha} \quad \text { and } \quad \lambda_{\alpha}, \mu_{\alpha} \rightarrow 1 .
$$

Let

$$
\phi_{\alpha}=\omega_{\xi_{\alpha}} \circ \pi_{\alpha} \text { and } \psi_{\alpha}=\omega_{\eta_{\alpha}} \circ \pi_{\alpha} .
$$

Since $\left\langle\xi_{\alpha}, \eta_{\alpha}\right\rangle=0$ we have $\left\langle\phi_{\alpha}, \psi_{\alpha}\right\rangle=0$. Passing to subnets if necessary, we may assume by compactness that $\phi_{\alpha} \rightarrow \phi$ and $\psi_{\alpha} \rightarrow \psi$ where $\phi, \psi \geq 0$ and $\|\phi\|,\|\psi\| \leq 1$. Since $\phi_{\alpha}(a)=\lambda_{\alpha} \rightarrow 1, \phi$ (and similarly $\psi)$ is a state.

Let $I$ be the kernel of the GNS representation $\pi_{\phi}$. Let $\sigma \in(A / I)^{\wedge}$; we claim $\pi_{\alpha} \rightarrow \sigma$. Let $K$ be a closed two-sided ideal of $A$ such that $\sigma(K) \neq\{0)$. Then $\phi(K) \neq\{0)$ and so there exists $\alpha_{0}$ such that $\phi_{\alpha}(K) \neq$ $\{0)$ and hence $\pi_{\alpha}(K) \neq\{0\}$ for all $\alpha \geq \alpha_{0}$. Thus $\pi_{\alpha} \rightarrow \sigma$ (see also [2, Lemma 3.8]). Hence

$$
(A / I)^{\wedge} \subseteq S(\pi)=(A / J)^{\wedge}
$$

and so $I \supseteq J$. Thus $\phi$ factors through $A / J$. Since $\pi$ is isolated in $(A / J)^{\wedge}$ and $\phi(a)=1, \phi=\rho \circ \pi$ where $\rho$ is the unique pure state of 
$\pi(A)$ supported by the rank one projection $\pi(a)$. The same applies to $\psi$ and so $\phi=\psi \in P(A)$. Thus $\left(\phi_{\alpha}, \psi_{\alpha}\right) \rightarrow(\phi, \phi)$ whereas $\left\langle\phi_{\alpha}, \psi_{\alpha}\right\rangle \rightarrow 0$. This contradicts the continuity of \langle\rangle and so $\delta$ and $U$ exist as claimed.

Since $\|\sigma(a)\| \rightarrow 1$ as $\sigma \rightarrow \pi$ we may assume, by shrinking $U$ if necessary, that $\|\sigma(a)\|>1-\delta / 2$ for $\sigma \in U$. Then for $\sigma \in U$ we have

$$
\sigma(a)=\|\sigma(a)\| e_{\sigma}+x_{\sigma}
$$

where $e_{\sigma}$ is a projection of rank one, $e_{\sigma} x_{\sigma}=x_{\sigma} e_{\sigma}=0$, and $\left\|x_{\sigma}\right\| \leq$ $1-\delta$. Define a function $f$ to be zero on $(-\infty, 1-\delta]$, one on $[1-\delta / 2, \infty)$ and linear on $[1-\delta, 1-\delta / 2]$. Then $f(a)$ is a positive element of $A$ and $\sigma(f(a))=e_{\sigma}$ for each $\sigma \in U$.

Recall that a $C^{*}$-algebra $A$ has continuous trace iff $\hat{A}$ is Hausdorff and $A$ satisfies Fell's condition: [4, 4.5.4]. Furthermore, $\hat{A}$ is Hausdorff iff the graph $R(A)$ of the unitary equivalence relation on $P(A)$ is closed in $P(A) \times P(A)[3,1.8 .3 .8]$. This combined with Theorem 2.3 gives us a characterization of continuous trace algebras in terms of $P(A)$ and $R(A)$.

Corollary 2.4. A $C^{*}$-algebra $A$ has continuous trace iff \langle\rangle is continuous for the product topology on $R(A)$ and $R(A)$ is closed in $P(A) \times$ $P(A)$.

Remarks. (1) Let $T: P(A) \times P(A) \rightarrow[0,1]$ be defined by $T(\phi, \psi)=$ $\langle\phi, \psi\rangle$. If $A$ has continuous trace, even if $A$ is abelian, $T$ need not be continuous on $P(A) \times P(A)$. In fact, continuity of $T$ on $P(A) \times P(A)$ is equivalent to $A$ being the $c_{0}$ direct sum of elementary $C^{*}$-algebras. For if $A$ is a $c_{0}$-sum $\bigoplus K_{\alpha}$ where each $K_{\alpha}$ is isomorphic to the algebra of compact operators on a Hilbert space $H_{\alpha}$, then $R(A)$ is the disjoint union of $P\left(K_{\alpha}\right) \times P\left(K_{\alpha}\right)$, and $T$ is continuous on $P\left(K_{\alpha}\right) \times P\left(K_{\alpha}\right)=$ $R\left(K_{\alpha}\right)$ by Theorem 2.3. On the other hand, if $T$ is continuous on $P(A) \times P(A)$, then $A$ is liminal by (the proof of) Theorem 2.3. Furthermore, $\hat{A}$ must be discrete: if $\pi_{\alpha} \rightarrow \pi$ in $\hat{A}$ with each $\pi_{\alpha}$ distinct from $\pi$, then by openness of the map $P(A) \rightarrow \hat{A}$ there is a net $\left\{\phi_{\beta}\right\}$ in $P(A)$ converging to some $\phi$ in $P(A)$ with no $\phi_{\beta}$ equivalent to $\phi$. Then each $\phi_{\beta}$ is orthogonal to $\phi$, and $\left(\phi_{\beta}, \phi\right) \rightarrow(\phi, \phi)$ in $P(A) \times P(A)$ shows $T$ is discontinuous on $P(A) \times P(A)$. It now follows that $A$ is the $c_{0}$-sum of liminal algebras $A_{\alpha}$ with each $\hat{A}_{\alpha}$ singleton. Thus each $A_{\alpha}$ is elementary, as claimed.

(2) It follows from Lemma 2.1 and the fact that the unit ball of $A^{*}$ is weak*-closed that $T$ is upper semi-continuous. Hence $T$ is automatically continuous at points $(\phi, \psi)$ for which $\langle\phi, \psi\rangle=0$. Thus $T$ 
is continuous on $P(A) \times P(A)$ if and only if $T$ is continuous at every point of $R(A)$. Of course, this can be quite different from continuity of $T$ restricted to $R(A)$, and the reader should keep in mind that we are using the symbol \langle\rangle to denote a map with domain $R(A)$.

(3) The requirement in Corollary 2.4 that $R(A)$ be closed in $P(A) \times$ $P(A)$ is not redundant, as can be seen by considering the algebra of continuous functions $f$ from $[0,1]$ into $M_{2}$ with $f(1)$ diagonal.

3. Transition probabilities and the quotient topology on $R(A)$. Recall from $\S 1$ that $G(A)$ is the set of extreme points of the unit ball of $A$, with the weak* topology. The quotient topology on $R(A)$ is that determined by the map $\phi \rightarrow\left(|\phi|,\left|\phi^{*}\right|\right)$ from $G(A)$ onto $R(A)$.

Each element $\phi$ of $G(A)$ can be viewed as a $\sigma$-weakly continuous functional on $A^{* *}$. In the lemma below, 1 is the identity of $A^{* *}$ (and of $A$, if $A$ has an identity).

LEMMA 3.1. The map $\phi \rightarrow \phi(1)$ is weak*-continuous on $G(A)$.

Proof. Suppose $\phi_{\alpha} \rightarrow \phi$ in $G(A)$. Choose irreducible representations $\left\{\pi_{\alpha}\right\}$ and $\pi$, and unit vectors $\left\{\xi_{\alpha}\right\},\left\{\eta_{\alpha}\right\}, \xi$, and $\eta$ such that for all $a$ in $A$,

$$
\phi_{\alpha}(a)=\left(\pi_{\alpha}(a) \xi_{\alpha}, \eta_{\alpha}\right), \quad \text { and } \quad \phi(a)=(\pi(a) \xi, \eta)
$$

Since $\phi_{\alpha} \rightarrow \phi$, then $\left|\phi_{\alpha}\right| \rightarrow|\phi|$, and so

$$
\left|\phi_{\alpha}\right|(a)=\left(\pi_{\alpha}(a) \xi_{\alpha}, \xi_{\alpha}\right) \rightarrow(\pi(a) \xi, \xi)=|\phi|(a) .
$$

Now fix $\varepsilon>0$ and choose $u$ in $a$ with $0 \leq u \leq 1$ and with $|\phi|(u)>$ $1-\varepsilon$. (Any approximate unit for $A$ converges $\sigma$-weakly in $A^{* *}$ to the identity element; let $u$ be a suitable member of this approximate unit.)

Note that

$$
\begin{aligned}
\|\pi(u) \xi-\xi\|^{2} & =\|\pi(1-u) \xi\|^{2}=\left(\pi(1-u)^{2} \xi, \xi\right) \\
& \leq(\pi(1-u) \xi, \xi)=|\phi|(1-u)<\varepsilon .
\end{aligned}
$$

Now for some $\alpha_{0}, \alpha \geq \alpha_{0}$ will imply that $\left|\phi_{\alpha}\right|(u)>1-\varepsilon$, and so as above

$$
\left\|\pi_{\alpha}(u) \xi_{\alpha}-\xi_{\alpha}\right\| \leq \sqrt{\varepsilon}, \quad \text { for all } \alpha \geq \alpha_{0} .
$$

Since $\phi_{\alpha} \rightarrow \phi$, then for some index $\alpha_{1}>\alpha_{0}$ and for all $\alpha \geq \alpha_{1}$ :

$$
\left|\phi_{\alpha}(u)-\phi(u)\right|=\left|\left(\pi_{\alpha}(u) \xi_{\alpha}, \eta_{\alpha}\right)-(\pi(u) \xi, \eta)\right|<\varepsilon .
$$

If we combine (4), (5), and (6) we get

$$
\left|\phi_{\alpha}(1)-\phi(1)\right|=\left|\left(\xi_{\alpha}, \eta_{\alpha}\right)-(\xi, \eta)\right|<\varepsilon+\sqrt{\varepsilon}+\sqrt{\varepsilon} \text { for } \alpha \geq \alpha_{1} \text {. }
$$

Since $\varepsilon$ was arbitrary, this shows $\phi_{\alpha}(1) \rightarrow \phi(1)$. 
Proposition 3.2. The map \langle\rangle on $R(A)$ is continuous for the quotient topology.

Proof. The composition of the quotient map from $G(A)$ onto $R(A)$ followed by the map \langle\rangle is given by

$$
\phi \rightarrow\left\langle|\phi|,\left|\phi^{*}\right|\right\rangle=|(\xi, \eta)|^{2}=|(\pi(1) \xi, \eta)|^{2}=|\phi(1)|^{2}
$$

where $\phi(a)=(\pi(a) \xi, \eta)$. By Lemma 3.1 this composition is continuous, and it follows that \langle\rangle is continuous for the quotient topology.

LEMMA 3.3. Let $\left\{\left(\phi_{\alpha}, \psi_{\alpha}\right)\right\}$ be a net in $R(A)$ which converges to $(\phi, \phi)$ for some $\phi$ in $P(A)$ for the product topology. Then $\left(\phi_{\alpha}, \psi_{\alpha}\right) \rightarrow$ $(\phi, \phi)$ in the quotient topology iff $\left\langle\phi_{\alpha}, \psi_{\alpha}\right\rangle \rightarrow 1$.

Proof. Continuity of \langle\rangle for the quotient topology proves the forward implication. For the converse, suppose $\left\langle\phi_{\alpha}, \psi_{\alpha}\right\rangle \rightarrow 1$. For each $\alpha$ choose a pure functional $\sigma_{\alpha}$ such that $\left|\sigma_{\alpha}\right|=\phi_{\alpha},\left|\sigma_{\alpha}^{*}\right|=\psi_{\alpha}$, and such that $\sigma_{\alpha}(1)$ is real and nonnegative. We are going to show that $\left\|\phi_{\alpha}-\sigma_{\alpha}\right\| \rightarrow 0$. For a fixed index $\alpha$, let $\pi$ be an irreducible representation of $A$ and $\xi$ and $\eta$ unit vectors such that

$$
\sigma_{\alpha}(x)=(\pi(x) \xi, \eta) \text { for all } x \text { in } A .
$$

Then

$$
\left|\sigma_{\alpha}\right|(x)=\phi_{\alpha}(x)=(\pi(x) \xi, \xi)
$$

and

$$
\begin{aligned}
\left|\phi_{\alpha}(x)-\sigma_{\alpha}(x)\right| & =|(\pi(x) \xi, \xi)-(\pi(x) \xi, \eta)| \\
& \leq\|x\|\|\xi-\eta\|=\|x\|(2-2 \operatorname{Re}(\xi, \eta))^{1 / 2} .
\end{aligned}
$$

Since we arranged that $\phi_{\alpha}(1)=(\xi, \eta)$ is real and nonnegative, then

$$
\left\langle\phi_{\alpha}, \psi_{\alpha}\right\rangle=|(\xi, \eta)|^{2}
$$

implies $\left\langle\phi_{\alpha}, \psi_{\alpha}\right\rangle^{1 / 2}=(\xi, \eta)$. Thus

$$
\left\|\phi_{\alpha}-\sigma_{\alpha}\right\| \leq\left(2-2\left\langle\phi_{\alpha}, \psi_{\alpha}\right\rangle^{1 / 2}\right)^{1 / 2}
$$

which approaches zero as $\left\langle\phi_{\alpha}, \psi_{\alpha}\right\rangle \rightarrow 1$. Then $\phi_{\alpha} \rightarrow \phi\left(\right.$ weak $\left.^{*}\right)$ implies $\sigma_{\alpha} \rightarrow \phi$, and now $\left|\sigma_{\alpha}\right|=\phi_{\alpha},\left|\sigma_{\alpha}^{*}\right|=\psi_{\alpha}$ implies that $\left(\phi_{\alpha}, \psi_{\alpha}\right) \rightarrow$ $\left(|\phi|,\left|\phi^{*}\right|\right)=(\phi, \phi)$ in the quotient topology. 
Proposition 3.4. The quotient and product topologies on $R(A)$ coincide iff transition probabilities are continuous on $R(A)$ for the product topology.

Proof. Let $\left(\phi_{\alpha}, \psi_{\alpha}\right) \rightarrow(\phi, \psi)$ in $R(A)$ for the product topology, and assume that \langle\rangle is continuous on $R(A)$ for the same topology. By Kadison's transitivity theorem, we can choose a unitary $u$ in $\tilde{A}$ such that $u^{*} \psi u=\phi$. Then $\left(\phi_{\alpha}, u^{*} \psi_{\alpha} u\right) \rightarrow(\phi, \phi)$ in the product topology, and for the quotient topology as well (by Lemma 3.3). By the openness of the map from $G(A)$ onto $G(A) / S^{1}$ (or by the proof of Lemma 3.3), there is a net $\left\{\sigma_{\beta}\right\}$ in $G(A)$ such that $\sigma_{\beta} \rightarrow \phi$ and such that the image of $\left\{\sigma_{\beta}\right\}$ in $R(A)$ is a subnet of $\left\{\left(\phi_{\alpha}, u^{*} \psi_{\alpha} u\right)\right\}$. (Thus $\left|\sigma_{\beta}\right|=\phi_{\beta}$ and $\left|\sigma_{\beta}^{*}\right|=u^{*} \psi_{\beta} u$.)

From the description of pure functionals in Proposition 1.1, it follows that multiplication of functionals in $A^{*}$ by a unitary in $\tilde{A}$ maps pure functionals to pure functionals. Furthermore, Proposition 1.1 gives the following results for absolute values:

$$
\begin{gathered}
\left|u \sigma_{\beta}\right|=\left|\sigma_{\beta}\right|=\phi_{\beta}, \\
\left|\left(u \sigma_{\beta}\right)^{*}\right|=u\left|\sigma_{\beta}^{*}\right| u^{*}=u\left(u^{*} \psi_{\beta} u\right) u^{*}=\psi_{\beta} .
\end{gathered}
$$

Thus $\left\{u \sigma_{\beta}\right\}$ is a net in $G(A)$ which converges to the pure functional $u \phi$, and the image under the quotient map $G(A) \rightarrow R(A)$ is a subnet $\left\{\left(\phi_{\beta}, \psi_{\beta}\right)\right\}$ of the original net $\left\{\left(\phi_{\alpha}, \psi_{\alpha}\right)\right\}$. Thus this subnet converges to $(\phi, \psi)$ in the quotient topology. Since this same argument can be applied to any subnet in place of $\left\{\left(\phi_{\alpha}, \psi_{\alpha}\right)\right\}$, it follows that $\left(\phi_{\alpha}, \psi_{\alpha}\right) \rightarrow$ $(\phi, \psi)$ in the quotient topology.

Conversely, suppose the quotient and product topologies coincide. Then \langle\rangle is continuous for both topologies by Proposition 3.2.

Note that in the proof of the reverse implication of Proposition 3.4, we only used continuity of \langle\rangle at points $(\phi, \phi)$ in the diagonal of $R(A)$. Thus we have the following consequence.

Corollary 3.5. Let $A$ be a $C^{*}$-algebra. Then \langle\rangle is continuous for the product topology on $R(A)$ iff \langle\rangle is continuous at each point in the diagonal of the equivalence relation $R(A)$.

The following rephrasing of the previous results was pointed out to us by Dana Williams and Jean Renault. 
Corollary 3.6. Let $A$ be a $C^{*}$-algebra. These are equivalent.

(i) $A$ has continuous trace.

(ii) The inclusion $R(A) \rightarrow P(A) \times P(A)$ is proper (where $R(A)$ has the quotient topology).

(iii) The canonical map from $G(A)$ into $P(A) \times P(A)$ is proper.

Proof. By [3, I.10.1, Prop. 2] the inclusion map $R(A) \rightarrow P(A) \times P(A)$ is proper iff (1) its image is closed and (2) it is a homeomorphism onto its image. The latter property is equivalent to continuity of \langle\rangle for the product topology by Proposition 3.4, and so equivalence of (i) and (ii) follows from Corollary 2.4. Since $R(A)$ is the quotient of $G(A)$ by the action of the compact group $S^{1}$, the map $G(A) \rightarrow R(A)$ is proper by [3, III.4.1.2]. By [3, I.10.1, Cor. 4] the map $G(A) \rightarrow P(A) \times P(A)$ will be proper iff $G(A) \rightarrow R(A)$ and $R(A) \rightarrow P(A) \times P(A)$ are proper, and this shows (iii) and (ii) are equivalent.

\section{REFERENCES}

[1] C. A. Akemann and B. Russo, Geometry of the unit sphere of a $C^{*}$-algebra and its dual, Pacific J. Math., 32 (1970), 575-585.

[2] C. A. Akemann and F. W. Shultz, Perfect $C^{*}$-algebras, Amer. Math. Soc., Memoir \#326, 1985.

[3] N. Bourbaki, General Topology, Hermann 1966.

[4] J. Dixmier, $C^{*}$-algebras, North Holland Publ. Co., 1977. (Translation of Les $C^{*}$-algèbres et leurs représentations, Gauthiers-Villars, Paris (1969).

[5] E. G. Effros, Order ideals in a $C^{*}$-algebra and its dual, Duke Math. J., 30 (1963), 391-412.

[6] J. Glimm, Type I $C^{*}$-algebras, Ann. of Math., 73 (1961), 572-612.

[7] A Stone-Weierstrass theorem for $C^{*}$-algebras, Ann. of Math., 72 (1960), 216-244.

[8] J. Glimm and R. V. Kadison, Unitary operators in $C^{*}$-algebras, Pacific J. Math., 10 (1960), 547-556.

[9] P. Green, $C^{*}$-algebras of transformation groups with smooth orbit space, Pacific J. Math., 72 (1977), 71-97.

[10] R. V. Kadison, Limits of states, Commun. Math. Phys., 85 (1982), 143-154.

[11] A. Kumjian, Preliminary algebras arising from local homeomorphisms, Math. Scand., 52 (1983), 269-278.

[12] R. T. Powers and E. Stormer, Free states of the canonical anti-commutation relations, Commun. Math. Phys., 16 (1970), 1-33.

[13] J. Renault, Two applications of the dual groupoid of a $C^{*}$-algebra, in Operator Algebras and their Connections with Topology and Ergodic Theory, Proceedings, Busteni, Romania 1983. Lecture Notes in Mathematics \#1132, Springer-Verlag.

[14] S. Sakai, $C^{*}$-algebras and $W^{*}$-algebras, Engebnisse der Mathematik und ihrer Grenzgebiete, Band 60, Springer-Verlag 1971.

[15] F. W. Shultz, Pure states as a dual object for $C^{*}$-algebras, Comun. Math. Phys., 82 (1982), 497-509. 
[16] E. Wigner, Gruppentheorie und Ihre Anwendung, Braunschweig: Vieweg 1931. English transl. by J. J. Griffin, New York: Academic Press 1959.

Received September 14, 1987. The second author was partially supported by NSF grant \# DMS-8201730.

UNIVERSITY OF ABERDEEN

THE EdWARd WRIGHT BUILDING

DUNBar ST.

ABERDEEN, AB9 2TY

AND

Wellesley College

WELLESLEY, MA 02181 



\section{PACIFIC JOURNAL OF MATHEMATICS EDITORS}

\author{
V. S. VARADARAJAN \\ (Managing Editor) \\ University of California \\ Los Angeles, CA 90024 \\ HeRbert Clemens \\ University of Utah \\ Salt Lake City, UT 84112 \\ THOMAS ENRIGHT \\ University of California, San Diego \\ La Jolla, CA 92093
}

R. FINN

Stanford University

Stanford, CA 94305

HERMANN FLASCHKA

University of Arizona

Tucson, AZ 85721

VAUGHAN F. R. JONES

University of California

Berkeley, CA 94720

STEVEN KERCKHOFF

Stanford University

Stanford, CA 94305

\section{ROBION KIRBY}

University of California

Berkeley, CA 94720

C. C. MOORE

University of California

Berkeley, CA 94720

HAROLD STARK

University of California, San Diego

La Jolla, CA 92093

\section{ASSOCIATE EDITORS}
R. ARenS
E. F. BECKENBACH
B. H. NeumanN
F. WOLF
K. YOSHIDA (1906-1982)

\section{SUPPORTING INSTITUTIONS}
UNIVERSITY OF ARIZONA
UNIVERSITY OF OREGON
UNIVERSITY OF BRITISH COLUMBIA
UNIVERSITY OF SOUTHERN CALIFORNIA
CALIFORNIA INSTITUTE OF TECHNOLOGY
STANFORD UNIVERSITY
UNIVERSITY OF CALIFORNIA
MONTANA STATE UNIVERSITY
UNIVERSITY OF HAWAII
UNIVERSITY OF NEVADA, RENO
UNIVERSITY OF TOKYO
NEW MEXICO STATE UNIVERSITY
UNIVERSITY OF UTAH
OREGON STATE UNIVERSITY
WASHINGTON STATE UNIVERSITY
UNIVERSITY OF WASHINGTON 


\section{Pacific Journal of Mathematics}

\section{Vol. 136, No. $1 \quad$ November, 1989}

Robert Archbold and Frederic W. Shultz, Characterization of $C^{*}$-algebras with continuous trace by properties of their pure states $\ldots \ldots \ldots \ldots \ldots \ldots 1$

Shu Ping Chen and Roberto Triggiani, Proof of extensions of two conjectures on structural damping for elastic systems $\ldots \ldots \ldots \ldots \ldots \ldots$

Philip Throop Church and James Timourian, A nonlinear elliptic

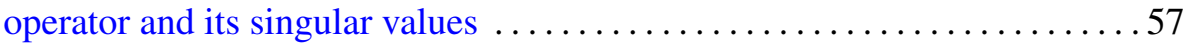

A. Gervasio Colares and Katsuei Kenmotsu, Isometric deformation of surfaces in $R^{3}$ preserving the mean curvature function $\ldots \ldots \ldots \ldots \ldots 71$

Fei Xu, A remark on spinor norms of local integral rotations. I . . . . . . . 81

Pedro Martinez Gadea and Ángel María Montesinos-Amilibia, Spaces of constant para-holomorphic sectional curvature $\ldots \ldots \ldots \ldots \ldots \ldots \ldots 5$

Guangxin Zeng, Homogeneous Stellensätze in semialgebraic geometry . . . . 103

Thomas Eric Hall, The isomorphism problem for orthodox semigroups . . . . 123

Mike Hoffman, Noncoincidence index, free group actions, and the fixed

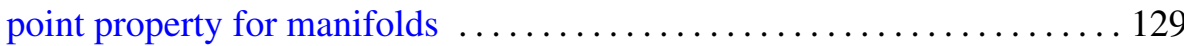

Terry Atherton Loring, The noncommutative topology of one-dimensional

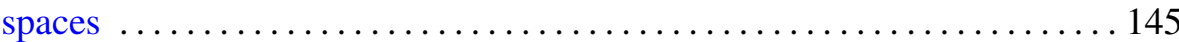

Haskell Paul Rosenthal and Alan Evan Wessel, The Krě̆ n-Mil'man property and a martingale coordinatization of certain nondentable convex sets

Yoshimi Saito, A remark on the limiting absorption principle for the reduced wave equation with two unbounded media 